\title{
Hitting the bullseye: endogenous electrophiles show remarkable nuance in signaling regulation
}

\author{
Marcus J. C. Long ${ }^{\ddagger}$, Pierre A. Miranda Herrera ${ }^{\dagger}$, and Yimon Aye ${ }^{\dagger *}$ \\ 'Swiss Federal Institute of Technology Lausanne (EPFL), 1015 Lausanne, Switzerland \\ ${ }^{+, \neq}$NCCR Chemical Biology and University of Geneva, 1211 Geneva, Switzerland \\ ‡University of Lausanne (UNIL), Lausanne, Switzerland
}

\begin{abstract}
Our bodies produce a host of electrophilic species that can label specific endogenous proteins in cells. The signaling roles of these molecules are underactive debate. However, in our opinion it is becoming increasingly likely that electrophiles can rewire cellular signaling processes at endogenous levels. Attention is turning more to understanding how nuanced electrophile signaling in cells is. In this perspective, we describe recent work from our laboratory that has started to inform on different levels of context-specific regulation of proteins by electrophiles. We discuss the relevance of these data to the field, and to the broader application of electrophile signaling to precision medicine development, beyond the traditional views of their pleiotropic cytotoxic roles.
\end{abstract}

*Correspondence yimon.aye@epfl.ch

Key words: electrophiles; signaling; profiling; drug mechanism; drug discovery; T-REX 
As we think everyone knows, life is not perfect. Indeed, life can be messy. The average American, for instance, produces 1700 pounds of garbage per year. It is unsurprising that managing waste production is an increasing problem in all aspects of society. Looking closer to our own workplaces, academic research generates 1000's of metric tons of hazardous waste per year in the US; a small fraction of what industry does ${ }^{1}$. Intriguingly, this aspect of our societal footprint runs contrary to the carefully crafted biochemical pathways that are often discussed as being the fabric of life as we understand it. Indeed, many a chemist is envious of the rapidity and cleanliness of day-to-day reactions that occur necessarily in our bodies. Extending the analogy a little, many a town planning officer would be very keen to emulate the number of intricate parts that the human body can organize and the enormous amounts of building and deconstruction that goes on inside each of us, ostensibly with relatively little waste.

But in fact, when we examine biological systems more closely, the sort of wasteful behavior we are accustomed to seeing in the wider world around us starts to crop up. For instance, 30 to $90 \%$ of all synthesized proteins are improper and directly degraded; an unusually futile cycle ${ }^{2}$. Even DNA synthesis is not immune from embedded error, with evidence for numerous severe replication errors that require homologous recombination to fix each cell division ${ }^{3}$. Even the exquisite chemical processes occurring in the mitochondria are not immune to these problems. Errant electrons can create radical species that are potentially chemically damaging ${ }^{4}$. Indeed, numerous reactive molecules so produced are at elevated and sustained levels toxic to cells and organisms.

All the above processes are hugely interesting and give insights into the fine line living systems walk between the (likely futile) pursuit of perfection, and the limitations of intrinsic biological processes. Our laboratory has principally invested its attention on how a specific type of reactive species, namely reactive electrophiles, behave in cells $s^{5-8}$. This work will be our focus here. It should be noted that, as the name implies, reactive electrophiles are inherently reactive products whose biology is only just coming to light. Indeed, outstanding questions in the field remain, including how relevant electrophile signaling is to endogenous signal regulation, and under what conditions electrophile signaling is most important. It is our objective in this paper to discuss recent findings from our work that shed new light on how reactive signals function on specific targets and propagate signals. These insights make a strong case for more nuanced ways of viewing reactive molecule signaling, which necessitate more precise means to interrogate sensor proteins. They further highlight that when an electrophile hits the right spot, i.e. is able to engage with a specific target protein in the correct specific cellular or subcellular context, interesting signaling processes can occur that have unexpected pathophysiological consequences. Such signaling changes can occur, even at low ligand occupancy, and in subcellular locales where the target proteins are not considered to be canonically active.

\section{Set your sights on the target}

Some years ago, the laboratory introduced REX technologies, G-REX and T-REX ${ }^{\mathrm{TM}}$ 6,9-11 (Figures 1A and B). T-REX method is a means to selectively target a specific protein with a given electrophile (native or otherwise) of choice in a living system. It transpires that T-REX ${ }^{\mathrm{TM}}$ works only on very electrophile sensitive proteins. Nevertheless, using T-REX ${ }^{\mathrm{TM}}$ we have validated multiple novel electrophile sensing proteins $(>15 \text {, so far })^{5,6,12-15}$. T-REX ${ }^{\mathrm{TM}}$ is also able to target specific proteins in specific subcellular environments with specific electrophiles as well as functioning in a range of model organisms ${ }^{12,16-20}$. T-REX ${ }^{\mathrm{TM}}$ has proven to be particularly insightful at assaying specific signaling consequences of targetspecific electrophile protein labeling 5,12,17,19,21-23. T-REX ${ }^{\mathrm{TM}}$ is a very precise method, which is both its greatest strength and source of uniqueness but it is also its ultimate weakness. Indeed, it is difficult to 
screen for protein sensors using T-REX ${ }^{\mathrm{TM}}$. To plug this gap, we later introduced G-REX ${ }^{\mathrm{TM}}{ }^{5}$. As a derivative of T-REX ${ }^{\mathrm{TM}}$, unsurprisingly, G-REX ${ }^{\mathrm{TM}}$ can also function in a locale-specific manner, and it can work in numerous model organisms (unpublished). Although both techniques work well independently, and can be combined with other methods, T-REX ${ }^{\mathrm{TM}}$ and G-REX ${ }^{\mathrm{TM}}$ work particularly well together.

Both these methods have allowed us to fix on specific targets, in specific locales or circumstances that are not usually possible in typical experiments using electrophiles ${ }^{16}$. Using these methods, we can also normalize electrophile dose, control timing of electrophile exposure, and bypass several slow steps in the uptake of electrophiles that have served to confound experiments carried out in living systems ${ }^{7,8}$. As REX ${ }^{\mathrm{TM}}$ methods will not be discussed in molecular detail here, we refer the curious reader to several published papers of ours for more information ${ }^{24}$ (Figure 1C). At the end of the manuscript, we will discuss why we think these new insights were achievable using REX ${ }^{\mathrm{TM}}$ technologies, as opposed to other methods.

\section{Who's in the firing line?}

We were intrigued by the approved multiple sclerosis drug Tecfidera ${ }^{\circledR 25,26}$, which is a highly reactive electrophile derived from fumarate (Figure 2A). Fumarate itself is a native electrophilic metabolite known to modulate several proteins, although fumarate's own role in biological signaling is likely minimal due to its slow labeling kinetics ${ }^{27}$. There were several models for how Tecfidera ${ }^{\circledR}$ functions, although in our opinion none were ideal, or at least had sufficient experimental backing ${ }^{28-38}$. One postulated target that was particularly interesting to us was Keap1, a known responder to electrophilic stress that we have shown is readily targetable under T-REX ${ }^{\mathrm{TM}}{ }^{6,9,10,15}$ One important issue with Keap1 being a target of Tecfidera ${ }^{\circledR}$ was that Tecfidera ${ }^{\circledR}$ is believed to suppress immune responses leading to remission of relapsing multiple sclerosis ${ }^{39}$. Unfortunately, Keap1's primary function upon electrophile labeling is stimulation of signaling responses that are overall cytoprotective ${ }^{40,41}$; little information was available pertaining to how Keap1 may function in a suppressive mechanism (Figure 3A).

We used Z-REX ${ }^{\mathrm{TM}} 8,12,19$, a derivative of T-REX ${ }^{\mathrm{TM}}$ performed in live zebrafish (Figure 2B), together with RNA-seq to examine how global mRNA pools were changed upon calibrated, sub-stoichiometric electrophile engagement of Keap1 in the vast majority of cells in zebrafish embryos ${ }^{19}$. Given that we were effectively performing the same process in each cell of the embryo, we were intrigued to find that under these conditions, selective loss in mRNAs specific to neutrophils and macrophages was observed $^{19}$. We proceeded to show that upon Z-REX ${ }^{\mathrm{TM}}$ targeting Keap1 in zebrafish and several mammalian cultured cells, upregulation of apoptosis occurs. Consistent with the RNA-seq data, the impact of targeting Keap1 is particularly elevated in innate immune cells, such as macrophages and neutrophils. Indeed, when we examined fish embryos that had undergone Z-REX ${ }^{\mathrm{TM}}$ targeting Keap1 which expressed fluorescent proteins within their macrophages or neutrophils, we showed selective depletion of these cells that was suppressed by caspase inhibitors ${ }^{19}$. This depletion did not happen when the same experiment was repeated, but the electrophile was not delivered to Keap1, or when a Keap1 mutant was used that cannot sense electrophiles ${ }^{19}$. Our further examination uncovered that Keap1 can bind to $\mathrm{Wdr} 1^{42,43}$, a protein linked to regulation of actin, and this binding is inhibited upon electrophile labeling of Keap $1^{19}$. Wdr1 released from Keap1 can interact with Cofilin ${ }^{42}$, which together trigger the mitochondrial apoptosis pathway, through $\mathrm{Bax}^{44}$, and ultimately triggering caspase signaling $^{19}$ (Figure 3B). Knockout of any of the proteins in this pathway suppressed the loss of neutrophils and macrophages caused by bolus Tecfidera ${ }^{\circledR}$ treatment in live zebrafish ${ }^{19}$. 
These data, particularly those derived using Z-REX ${ }^{\mathrm{TM}}$ indicate that different cells' responses to similar levels of electrophilic engagement of the same protein are unequal. Indeed, we have shown that Keap1 targeting in whole fish under Z-REX ${ }^{\mathrm{TM}}$ does stimulate cytoprotective responses as expected ${ }^{19}$. However, in neutrophils and macrophages, an unexpected response occurs. This unexpected response is effectively the inverse of the expected outcome, cell death. Since Z-REX ${ }^{\mathrm{TM}}$ is calibrated in terms of expression, and has been shown to give similar labeling of the target protein in different situations, the predominance of data point to substantial rewiring of how neutrophils and macrophages respond to electrophiles. Although this finding raises new questions, principally why would such a sensitization to Wdr1 level elevation occur, we stress that (1) such an outcome was not predictable based on data available prior to our experiments, and (2) Wdr1's role in immune cells in general is established, although its precise role and links to viability are less clear.

\section{Where did the shooting start?}

In another recent paper ${ }^{45}$, we used G-REX ${ }^{\mathrm{TM}}$ to release the same electrophile at ostensibly similar concentrations in either the nucleus or the cytosol and profile proteins that were labelled selectively by the electrophile only in one or the other locale (Figure 4A and B). This specific technique cannot identify proteins that sense electrophiles equally in both locales; we further note that primary data from this screen cannot in of themselves prove that a specific protein is more sensitive in one locale than another, as this could be dominated by intrinsic localization preferences. Nevertheless, several proteins that were putative locale-specific electrophile sensors were unearthed using this method. Rewardingly, the vast majority were validated for subcellular locale specific sensing using a variant of T-REX ${ }^{\mathrm{TM}}$ (Localis-REX) and bolus dosing. In both these examples proteins were localized to the nucleus or cytosol using specific localization tags and their intrinsic electrophile sensitivities were examined.

One of the most intriguing proteins to emerge from this screen was CDK9 (Figure 5A and B). This protein is canonically a nuclear kinase that is a critical component of the positive transcription elongation factor complex, P-TEFb, along with CyclinT1, another nuclear localized protein ${ }^{46,47}$. The PTEFb complex is essential for promoting transcription by releasing RNA polymerase 2 from promoter proximal pause sites ${ }^{48,49}$. Intriguingly in our screen, and also during validation, CDK9 emerged to be an electrophile sensor only when present in the cytosol: nuclear CDK9 was not able to sense electrophiles well, although some electrophile sensing was observed.

\section{Who was around at the time of the shooting?}

We investigated if the relative recalcitrance of CDK9 to electrophile sensing in the nucleus was due to specific associating partners. Knockdown of CyclinT1 or a negative regulator of CDK9 that is also nuclear, HEXIM1 ${ }^{50,51}$, led to increased electrophile sensing by CDK9 in the nucleus (Figure 6). This result as well as the discovery of nuclear-specific electrophile sensors showed that the nucleus is permissive to electrophile sensing, but further demonstrates that electrophile sensing can be modulated by the target protein's interactome. Although we and indeed others have not investigated this link thoroughly, this implies that electrophile sensitivity could offer insight into associated proteomes, likely in a function dependent manner.

\section{Was the target moved?}


Given CDK9's strong links to transcriptional regulation, we postulated that CDK9 electrophile labeling may lead to changes in transcriptional activity. Indeed, electrophile labeling of wt-CDK9 elicited a loss of RNA polymerase 2 activity. This was however not seen in either CDK9 bearing a nuclear localization tag, or CDK9 bearing a nuclear exclusion sequence. These data imply that although CDK9 senses electrophiles well in the cytosol, the electrophile-modified CDK9 needs to move back into the nucleus in order to effect changes in the activity of the unmodified protein (Figure 6).

This result is indeed the first example of an electrophile-modified protein behaving as a long-distance signal in its own right. In previous literature we have discussed how for electrophiles to signal, the target protein needs to be not only acutely tuned to react with the electrophile but also must undergo activity or function modulations induced upon labeling by the electrophile. We have postulated that these two properties may, in some instances, be synergistic. Such an effect could possibly occur through the presence of a binding site for $\mathrm{HNE}^{52}$. Indeed, the proposed transience of electrophilic signals under the highly reducing and xenobiotic metabolizing power of the cell, could lead to a buildup of modified proteins in a position where they could not signal. Under such a situation, as electrophile labeling is irreversible, the modified protein would have to ferry the information contained by the electrophile to the intended target ${ }^{53}$.

\section{Beyond the blunderbuss effect?}

From the above discussion, and indeed by perusal of some of our previous work, it should be clear that electrophile sensing is surprisingly nuanced. Indeed, the above data shows signaling processes overall strongly reminiscent of canonical signaling pathways, albeit with relatively few key players (note we are dealing with signaling events that effectively cannot interchange between different proteins). Perhaps the most important take home message is the critical aspect of context to electrophile signaling that has been appreciated in some areas for some time, but has rarely been directly observed, until recently ${ }^{54}$. We would like to suggest that the principal reason for this lag is the fact that traditional methods to study electrophile signaling used methods exclusively reliant on bolus dosing to identify electrophile reactive proteins ${ }^{24}$. This strategy could readily be referred to as the blunderbuss approach, named after an early shotgun capable of spraying projectiles over a large area ${ }^{55}$ (Figure 7A). It involves using typically a large excess of electrophiles and reading out proteins that are labelled after prolonged time. Of course, this method has the habit of hitting many proteins nonspecifically, and can also change redox status of cells when carried out in living systems, although this is frequently performed in lysates. Thus, proteins identified as sensors can be dominated by abundant proteins, those with high cysteine content, those that are stable under the reaction conditions. As the conditions are harsh, they can also mask very reactive proteins whose sensing capabilities may require the cell to retain a balanced reducing environment.

Indeed, bolus dosing is leveraged in numerous modern methods to investigate electrophile signaling, for instance the various derivatives of activity-based protein profiling (ABPP) and other variants that have come after it $\mathrm{it}^{24,56,57}$. These methods are uniquely able to quantify absolute occupancy of a specific electrophilic ligand on a specific cysteine (or other) residue, and do to some extent get around the blunderbuss issue. These methods have even shown that even under excess doses the number of proteins that can attain high electrophile load is relatively low ${ }^{58}$. However, a lot of the proteins we have described as electrophile sensors are not scored in sensors in ABPP assays, either because they are not present among the proteins profiled (a common weakness of this method) or because they cannot attain a threshold labeling occupancy to score as a priority hit. Conventional thinking states that if a protein is sensitive to electrophiles, i.e. that it is labelled rapidly by an electrophile, then it will 
necessarily obtain a high electrophile labeling load and show up as a hit in ABPP, provided it can be detected. I.e. sensors are ranked based on ability to rapidly attain high occupancy by a specific electrophile. However, our data predict such a criterion is not necessarily a good metric for assigning electrophile responsivity. Indeed, some proteins could readily obtain fractional occupancy rapidly, reflecting a subset of electrophile responsive forms of the protein in question, for instance in a specific locale, or potentially a post translationally modified state. The remaining fraction(s) of the protein could then be inert to labeling by the electrophile of choice. Indeed, the activity profiling aspect of ABPP (where cells are treated with a highly reactive electrophile to assess modification of specific cysteines relative to a control sample) is often carried out post cell lysis using a highly reactive electrophilic species. Thus, it is quite possible that some of the internal cellular regulation we have found could be lost, or even that the internal regulation to relatively low reactivity native electrophiles is lost relative to the probe used in ABPP. These concerns are even more elevated when electrophile engagement in whole organisms is considered.

One of the key mitigating factors for the heavy reliance on bolus dosing is the simplicity of electrophiles and the fact that we do not really have a particularly good idea of how electrophile behave in cells, or even how much electrophile there is in specific regions of cells (or the whole cell often $)^{59}$. Evidence exists that electrophiles have a relatively short half-life in cellular environments, but this does little to build up a very nuanced picture ${ }^{60}$, as in addition to other unknowns, we do not know what concentrations of electrophiles are needed to signal (although our work suggests that this can be close to the concentration of several endogenous proteins, provided the protein is in the correct place at the correct time $\left.{ }^{61,62}\right)$. Some evidence has been proposed that electrophile diffusion distances are short, but how relevant localization of the signal, or diffusion of electrophile/modified protein are to signaling has remained untestable. With the data from G-REX ${ }^{\mathrm{TM}}$ screens above, where a transient amount of electrophile is released in specific locales, we do indeed start to build up a picture that HNE is certainly able to exert its labeling effects principally on the locale to which it was confined (hits from locale specific screening were for the overwhelming number of cases correct, for instance). Thus, we believe that several lines of evidence push for a movement towards more precision methods to interrogate electrophile signaling.

\section{So what if we follow this trajectory?}

We have outlined what we currently understand about nuanced electrophile signaling modes. We have stressed context is crucially important, giving examples of antagonistic cell-specific response effects and also locale-specific electrophile sensing, followed by translocation events that are necessary for signaling. We do not believe these events are likely to have arisen by chance. Evidence for this can be seen in the strong conservation of electrophilic sensing residues, for instance across Akt3 isoforms ${ }^{63}$. We further do not believe that the examples we have uncovered are likely to be isolated, given that we have begun only to scratch the surface of the panoply of tissue specific and locale specific signaling events that could be conceived. This means that electrophiles are likely important players in cellular signaling and hence decision making, in a cell-type-, or locale- (or both) dependent manner. Considering how disease states can rewire production of electrophilic stressors ${ }^{64}$, and indeed change protein locales ${ }^{65}$ we could readily extend this postulate to disease states, which could have novel electrophile responsivities reflective of changed protein expression, localization, or partnering. In genetic diseases, or cancer ${ }^{66}$, environmental effects or mutations ${ }^{67}$ also could readily serve to promote such changes. Indeed, changes in subcellular localization of important metabolites ${ }^{68}$, and proteins is increasingly linked to disease and (aberrant) signaling; gleaning such information is 
clearly important, but gaining understanding of locale-specific function and its relation to cell signaling is equally important ${ }^{69}$.

\section{A magic bullet?}

Given the above data, and the ensuing reasoning, we propose that mapping nuanced electrophile responsivity changes, especially those that occur across specific cell types, or specific disease states could serve as a pathway to uncovering novel associations, signaling modes, or pathway modes. In classical biology, unearthing such data are hugely important and are usually derived through some sort of high throughput/content/proteomewide strategy (e.g. G-REX ${ }^{\mathrm{TM}}$ ). However, follow up on such hits can be laborious as traditional biochemical/cell biology experiments need to be performed. Particularly in terms of context-specific effects (in subcellular locale or in specific tissues) chemical or genetic actionability of specific states of individual proteins or specific moonlighting roles require huge investments of time to perform with correct controls ${ }^{70}$. The pathways to examining druggability, should this be even on the table, are even less direct, and unlikely to be able to target a specific locale of a protein, should this be important for drug action.

However, electrophile-responsive pathways are different. Once electrophile sensing is discovered, TREX $^{\mathrm{TM}}$ can be immediately used to modulate and interrogate signaling and function, and elucidate downstream pathways directly (Figure 7B). As we have shown, this can be performed in specific locales. Further down the line, we have shown that specific inhibitors, or potentially modulators can be derived using standard medicinal chemistry steps ${ }^{71}$. There is no reason to assume this process could not be used to target specific subforms of a protein (for instance those in a specific locale sensitive to a specific electrophile) should that exist. Thus, in the electrophile signaling landscape, discovering novel signaling nodes necessarily informs on precision chemical actionability. This stands in contrast to traditional genetic screens which offer little insight in this area. Indeed, each electrophile sensitive protein identified offers access to (context-dependent) target modulation. This can be either inhibitory, or stimulatory, or potentially gain of function ${ }^{72}$. We have identified proteins that sense electrophiles through T-REX ${ }^{\mathrm{TM}}$ but do not seem to respond functionally to electrophiles ${ }^{73}$. These for the time being appear to be proteins that are only capable of achieving very low occupancy under standard T-REX ${ }^{\mathrm{TM}}$ methods. However, we cannot exclude the possibility that electrophile labeling affects a property of the proteins hitherto unknown. Achieving at least approximate means to triage hits from T-REX ${ }^{\mathrm{TM}}$ or G-REX ${ }^{\mathrm{TM}}$ screens to focus on the most relevant sensors is one goal of our laboratory. Nevertheless, we believe that our data are starting to open new possibilities in the field of biology and medicinal chemistry that are beyond the reach of traditional activity probes and drugs. Time will tell if we are on target with this prediction, or not. 


\section{References}

(1) United States., O. of S. Waste. Report to Congress : Management of Hazardous Wastes from Educational Institutions; The Office: Washington, D.C., 1989.

(2) Rothman, S. How Is the Balance between Protein Synthesis and Degradation Achieved? Theor. Biol. Med. Model. 2010, 7 (1), 1-11.

(3) Claussin, C.; Porubský, D.; Spierings, D. C.; Halsema, N.; Rentas, S.; Guryev, V.; Lansdorp, P. M.; Chang, M. Genome-Wide Mapping of Sister Chromatid Exchange Events in Single Yeast Cells Using Strand-Seq. eLife 2017, 6, e30560. https://doi.org/10.7554/eLife.30560.

(4) Cadenas, E.; Davies, K. J. A. Mitochondrial Free Radical Generation, Oxidative Stress, and Aging11This Article Is Dedicated to the Memory of Our Dear Friend, Colleague, and Mentor Lars Ernster (1920-1998), in Gratitude for All He Gave to Us. Free Radic. Biol. Med. 2000, 29 (3), 222-230. https://doi.org/10.1016/S0891-5849(00)00317-8.

(5) Zhao, Y.; Long, M. J. C.; Wang, Y.; Zhang, S.; Aye, Y. Ube2V2 Is a Rosetta Stone Bridging Redox and Ubiquitin Codes, Coordinating DNA Damage Responses. ACS Cent. Sci. 2018, 4 (2), 246259. https://doi.org/10.1021/acscentsci.7b00556.

(6) Fang, X.; Fu, Y.; Long, M. J. C.; Haegele, J. A.; Ge, E. J.; Parvez, S.; Aye, Y. Temporally Controlled Targeting of 4-Hydroxynonenal to Specific Proteins in Living Cells. J. Am. Chem. Soc. 2013, 135 (39), 14496-14499. https://doi.org/10.1021/ja405400k.

(7) Parvez, S.; Long, M. J. C.; Poganik, J. R.; Aye, Y. Redox Signaling by Reactive Electrophiles and Oxidants. Chem. Rev. 2018, 118 (18), 8798-8888.

https://doi.org/10.1021/acs.chemrev.7b00698.

(8) Long, M. J. C.; Rogg, C.; Aye, Y. An Oculus to Profile and Probe Target Engagement In Vivo: How T-REX Was Born and Its Evolution into G-REX. Acc. Chem. Res. 2021, 54 (3), 618-631. https://doi.org/10.1021/acs.accounts.0c00537.

(9) Parvez, S.; Fu, Y.; Li, J.; Long, M. J. C.; Lin, H.-Y.; Lee, D. K.; Hu, G. S.; Aye, Y. Substoichiometric Hydroxynonenylation of a Single Protein Recapitulates Whole-Cell-Stimulated Antioxidant Response. J. Am. Chem. Soc. 2015, 137 (1), 10-13. https://doi.org/10.1021/ja5084249.

(10) Lin, H.-Y.; Haegele, J. A.; Disare, M. T.; Lin, Q.; Aye, Y. A Generalizable Platform for Interrogating Target- and Signal-Specific Consequences of Electrophilic Modifications in Redox-Dependent Cell Signaling. J. Am. Chem. Soc. 2015, 137 (19), 6232-6244. https://doi.org/10.1021/ja5132648.

(11) Parvez, S.; Long, M. J. C.; Lin, H.-Y.; Zhao, Y.; Haegele, J. A.; Pham, V. N.; Lee, D. K.; Aye, Y. TREX on-Demand Redox Targeting in Live Cells. Nat. Protoc. 2016, 11 (12), 2328-2356. https://doi.org/10.1038/nprot.2016.114.

(12) Long, M. J. C.; Parvez, S.; Zhao, Y.; Surya, S. L.; Wang, Y.; Zhang, S.; Aye, Y. Akt3 Is a Privileged First Responder in Isozyme-Specific Electrophile Response. Nat. Chem. Biol. 2017, 13 (3), 333338. https://doi.org/10.1038/nchembio.2284.

(13) Liu, X.; Long, M. J. C.; Hopkins, B. D.; Luo, C.; Wang, L.; Aye, Y. Precision Targeting of Pten-Null Triple-Negative Breast Tumors Guided by Electrophilic Metabolite Sensing. ACS Cent. Sci. 2020, 6 (6), 892-902. https://doi.org/10.1021/acscentsci.9b00893.

(14) Surya, S. L.; Long, M. J. C.; Urul, D. A.; Zhao, Y.; Mercer, E. J.; Elsaid, I. M.; Evans, T.; Aye, Y. Cardiovascular Small Heat Shock Protein HSPB7 Is a Kinetically Privileged Reactive Electrophilic Species (RES) Sensor. ACS Chem. Biol. 2018, 13 (7), 1824-1831. https://doi.org/10.1021/acschembio.7b00925.

(15) Long, M. J.; Lin, H.-Y.; Parvez, S.; Zhao, Y.; Poganik, J. R.; Huang, P.; Aye, Y. $\beta$-TrCP1 Is a Vacillatory Regulator of Wnt Signaling. Cell Chem. Biol. 2017, 24 (8), 944-957.e7. https://doi.org/10.1016/j.chembiol.2017.06.009.

(16) C. Long, M. J.; Zhao, Y.; Aye, Y. Neighborhood Watch: Tools for Defining Locale-Dependent Subproteomes and Their Contextual Signaling Activities. RSC Chem. Biol. 2020, 1 (2), 42-55. https://doi.org/10.1039/DOCB00041H. 
(17) Long, M. J. C.; Urul, D. A.; Aye, Y. Chapter Eleven - REX Technologies for Profiling and Decoding the Electrophile Signaling Axes Mediated by Rosetta Stone Proteins. In Methods in Enzymology; Shukla, A. K., Ed.; Chemical and Synthetic Biology Approaches To Understand Cellular Functions - Part C; Academic Press, 2020; Vol. 633, pp 203-230. https://doi.org/10.1016/bs.mie.2019.02.039.

(18) Long, M. J. C.; Poganik, J. R.; Ghosh, S.; Aye, Y. Subcellular Redox Targeting: Bridging in Vitro and in Vivo Chemical Biology. ACS Chem. Biol. 2017, 12 (3), 586-600. https://doi.org/10.1021/acschembio.6b01148.

(19) Poganik, J. R.; Huang, K.-T.; Parvez, S.; Zhao, Y.; Raja, S.; Long, M. J. C.; Aye, Y. Wdr1 and Cofilin Are Necessary Mediators of Immune-Cell-Specific Apoptosis Triggered by Tecfidera. Nat. Commun. 2021, 12 (1), 5736. https://doi.org/10.1038/s41467-021-25466-x.

(20) Long, M. J. C.; Urul, D. A.; Chawla, S.; Lin, H.-Y.; Zhao, Y.; Haegele, J. A.; Wang, Y.; Aye, Y. Precision Electrophile Tagging in Caenorhabditis Elegans. Biochemistry 2018, 57 (2), 216-220. https://doi.org/10.1021/acs.biochem.7b00642.

(21) Liu, X.; Long, M. J. C.; Aye, Y. Proteomics and Beyond: Cell Decision-Making Shaped by Reactive Electrophiles. Trends Biochem. Sci. 2019, 44 (1), 75-89. https://doi.org/10.1016/j.tibs.2018.09.014.

(22) Long, M. J. C.; Aye, Y. The Die Is Cast: Precision Electrophilic Modifications Contribute to Cellular Decision Making. Chem. Res. Toxicol. 2016, 29 (10), 1575-1582. https://doi.org/10.1021/acs.chemrestox.6b00261.

(23) Poganik, J. R.; Long, M. J. C.; Aye, Y. Getting the Message? Native Reactive Electrophiles Pass Two Out of Three Thresholds to Be Bona Fide Signaling Mediators. BioEssays 2018, 40 (5), 1700240. https://doi.org/10.1002/bies.201700240.

(24) Parvez, S.; Long, M. J. C.; Lin, H.-Y.; Zhao, Y.; Haegele, J. A.; Pham, V. N.; Lee, D. K.; Aye, Y. TREX on-Demand Redox Targeting in Live Cells. Nat. Protoc. 2016, 11 (12), 2328-2356. https://doi.org/10.1038/nprot.2016.114.

(25) Fox, R. J.; Miller, D. H.; Phillips, J. T.; Hutchinson, M.; Havrdova, E.; Kita, M.; Yang, M.; Raghupathi, K.; Novas, M.; Sweetser, M. T.; Viglietta, V.; Dawson, K. T. Placebo-Controlled Phase 3 Study of Oral BG-12 or Glatiramer in Multiple Sclerosis. N. Engl. J. Med. 2012, 367 (12), 1087-1097. https://doi.org/10.1056/NEJMoa1206328.

(26) Gold, R.; Kappos, L.; Arnold, D. L.; Bar-Or, A.; Giovannoni, G.; Selmaj, K.; Tornatore, C.; Sweetser, M. T.; Yang, M.; Sheikh, S. I.; Dawson, K. T. Placebo-Controlled Phase 3 Study of Oral BG-12 for Relapsing Multiple Sclerosis. N. Engl. J. Med. 2012, 367 (12), 1098-1107. https://doi.org/10.1056/NEJMoa1114287.

(27) Jackson, P. A.; Widen, J. C.; Harki, D. A.; Brummond, K. M. Covalent Modifiers: A Chemical Perspective on the Reactivity of $\alpha, \beta$-Unsaturated Carbonyls with Thiols via Hetero-Michael Addition Reactions. J. Med. Chem. 2017, 60 (3), 839-885. https://doi.org/10.1021/acs.jmedchem.6b00788.

(28) Linker, R. A.; Lee, D.-H.; Ryan, S.; van Dam, A. M.; Conrad, R.; Bista, P.; Zeng, W.; Hronowsky, X.; Buko, A.; Chollate, S.; Ellrichmann, G.; Brück, W.; Dawson, K.; Goelz, S.; Wiese, S.; Scannevin, R. H.; Lukashev, M.; Gold, R. Fumaric Acid Esters Exert Neuroprotective Effects in Neuroinflammation via Activation of the Nrf2 Antioxidant Pathway. Brain 2011, 134 (3), 678692. https://doi.org/10.1093/brain/awq386.

(29) Gopal, S.; Mikulskis, A.; Gold, R.; Fox, R. J.; Dawson, K. T.; Amaravadi, L. Evidence of Activation of the Nrf2 Pathway in Multiple Sclerosis Patients Treated with Delayed-Release Dimethyl Fumarate in the Phase 3 DEFINE and CONFIRM Studies. Mult. Scler. J. 2017, 23 (14), 18751883. https://doi.org/10.1177/1352458517690617.

(30) Andersen, J. L.; Gesser, B.; Funder, E. D.; Nielsen, C. J. F.; Gotfred-Rasmussen, H.; Rasmussen, M. K.; Toth, R.; Gothelf, K. V.; Arthur, J. S. C.; Iversen, L.; Nissen, P. Dimethyl Fumarate Is an Allosteric Covalent Inhibitor of the P90 Ribosomal S6 Kinases. Nat. Commun. 2018, 9 (1), 4344. https://doi.org/10.1038/s41467-018-06787-w. 
(31) Gesser, B.; Rasmussen, M. K.; Raaby, L.; Rosada, C.; Johansen, C.; Kjellerup, R. B.; Kragballe, K.; Iversen, L. Dimethylfumarate Inhibits MIF-Induced Proliferation of Keratinocytes by Inhibiting MSK1 and RSK1 Activation and by Inducing Nuclear p-c-Jun (S63) and p-P53 (S15) Expression. Inflamm. Res. 2011, 60 (7), 643-653. https://doi.org/10.1007/s00011-011-03167.

(32) Kastrati, I.; Siklos, M. I.; Calderon-Gierszal, E. L.; El-Shennawy, L.; Georgieva, G.; Thayer, E. N.; Thatcher, G. R. J.; Frasor, J. Dimethyl Fumarate Inhibits the Nuclear Factor KB Pathway in Breast Cancer Cells by Covalent Modification of P65 Protein *. J. Biol. Chem. 2016, 291 (7), 3639-3647. https://doi.org/10.1074/jbc.M115.679704.

(33) Schulze-Topphoff, U.; Varrin-Doyer, M.; Pekarek, K.; Spencer, C. M.; Shetty, A.; Sagan, S. A.; Cree, B. A. C.; Sobel, R. A.; Wipke, B. T.; Steinman, L.; Scannevin, R. H.; Zamvil, S. S. Dimethyl Fumarate Treatment Induces Adaptive and Innate Immune Modulation Independent of Nrf2. Proc. Natl. Acad. Sci. 2016, 113 (17), 4777-4782. https://doi.org/10.1073/pnas.1603907113.

(34) Mrowietz, U.; Morrison, P. J.; Suhrkamp, I.; Kumanova, M.; Clement, B. The Pharmacokinetics of Fumaric Acid Esters Reveal Their In Vivo Effects. Trends Pharmacol. Sci. 2018, 39 (1), 1-12. https://doi.org/10.1016/j.tips.2017.11.002.

(35) Blewett, M. M.; Xie, J.; Zaro, B. W.; Backus, K. M.; Altman, A.; Teijaro, J. R.; Cravatt, B. F. Chemical Proteomic Map of Dimethyl Fumarate-Sensitive Cysteines in Primary Human T Cells. Sci. Signal. 2016. https://doi.org/10.1126/scisignal.aaf7694.

(36) Blatnik, M.; Frizzell, N.; Thorpe, S. R.; Baynes, J. W. Inactivation of Glyceraldehyde-3Phosphate Dehydrogenase by Fumarate in Diabetes: Formation of S-(2-Succinyl)Cysteine, a Novel Chemical Modification of Protein and Possible Biomarker of Mitochondrial Stress. Diabetes 2008, 57 (1), 41-49. https://doi.org/10.2337/db07-0838.

(37) Chen, H.; Assmann, J. C.; Krenz, A.; Rahman, M.; Grimm, M.; Karsten, C. M.; Köhl, J.; Offermanns, S.; Wettschureck, N.; Schwaninger, M. Hydroxycarboxylic Acid Receptor 2 Mediates Dimethyl Fumarate's Protective Effect in EAE. J. Clin. Invest. 2014, 124 (5), 21882192. https://doi.org/10.1172/JCI72151.

(38) Gillard, G. O.; Collette, B.; Anderson, J.; Chao, J.; Scannevin, R. H.; Huss, D. J.; Fontenot, J. D. DMF, but Not Other Fumarates, Inhibits NF-KB Activity in Vitro in an Nrf2-Independent Manner. J. Neuroimmunol. 2015, 283, 74-85. https://doi.org/10.1016/j.jneuroim.2015.04.006.

(39) Poganik, J. R.; Aye, Y. Electrophile Signaling and Emerging Immuno- and Neuro-Modulatory Electrophilic Pharmaceuticals. Front. Aging Neurosci. 2020, 12, 1. https://doi.org/10.3389/fnagi.2020.00001.

(40) Baird, L.; Dinkova-Kostova, A. T. The Cytoprotective Role of the Keap1-Nrf2 Pathway. Arch. Toxicol. 2011, 85 (4), 241-272. https://doi.org/10.1007/s00204-011-0674-5.

(41) Kansanen, E.; Kuosmanen, S. M.; Leinonen, H.; Levonen, A.-L. The Keap1-Nrf2 Pathway: Mechanisms of Activation and Dysregulation in Cancer. Redox Biol. 2013, 1 (1), 45-49. https://doi.org/10.1016/j.redox.2012.10.001.

(42) Li, J.; Brieher, W. M.; Scimone, M. L.; Kang, S. J.; Zhu, H.; Yin, H.; von Andrian, U. H.; Mitchison, T.; Yuan, J. Caspase-11 Regulates Cell Migration by Promoting Aip1-Cofilin-Mediated Actin Depolymerization. Nat. Cell Biol. 2007, 9 (3), 276-286. https://doi.org/10.1038/ncb1541.

(43) Kuhns, D. B.; Fink, D. L.; Choi, U.; Sweeney, C.; Lau, K.; Priel, D. L.; Riva, D.; Mendez, L.; Uzel, G.; Freeman, A. F.; Olivier, K. N.; Anderson, V. L.; Currens, R.; Mackley, V.; Kang, A.; Al-Adeli, M.; Mace, E.; Orange, J. S.; Kang, E.; Lockett, S. J.; Chen, D.; Steinbach, P. J.; Hsu, A. P.; Zarember, K. A.; Malech, H. L.; Gallin, J. I.; Holland, S. M. Cytoskeletal Abnormalities and Neutrophil Dysfunction in WDR1 Deficiency. Blood 2016, 128 (17), 2135-2143. https://doi.org/10.1182/blood-2016-03-706028.

(44) Wei, M. C.; Zong, W.-X.; Cheng, E. H.-Y.; Lindsten, T.; Panoutsakopoulou, V.; Ross, A. J.; Roth, K. A.; MacGregor, G. R.; Thompson, C. B.; Korsmeyer, S. J. Proapoptotic BAX and BAK: A 
Requisite Gateway to Mitochondrial Dysfunction and Death. Science 2001. https://doi.org/10.1126/science.1059108.

(45) Zhao, Y.; Miranda Herrera, P. A.; Chang, D.; Hamelin, R.; Long, M. J. C.; Aye, Y. FunctionGuided Proximity Mapping Unveils Electrophilic-Metabolite Sensing by Proteins Not Present in Their Canonical Locales. Proc. Natl. Acad. Sci. 2022, 119 (5), e2120687119. https://doi.org/10.1073/pnas.2120687119.

(46) Peterlin, B. M.; Price, D. H. Controlling the Elongation Phase of Transcription with P-TEFb. Mol. Cell 2006, 23 (3), 297-305. https://doi.org/10.1016/j.molcel.2006.06.014.

(47) Price, D. H. P-TEFb, a Cyclin-Dependent Kinase Controlling Elongation by RNA Polymerase II. Mol. Cell. Biol. 2000. https://doi.org/10.1128/MCB.20.8.2629-2634.2000.

(48) Jonkers, I.; Lis, J. T. Getting up to Speed with Transcription Elongation by RNA Polymerase II. Nat. Rev. Mol. Cell Biol. 2015, 16 (3), 167-177. https://doi.org/10.1038/nrm3953.

(49) Zhou, Q.; Li, T.; Price, D. H. RNA Polymerase II Elongation Control. Annu. Rev. Biochem. 2012, 81 (1), 119-143. https://doi.org/10.1146/annurev-biochem-052610-095910.

(50) Kobbi, L.; Demey-Thomas, E.; Braye, F.; Proux, F.; Kolesnikova, O.; Vinh, J.; Poterszman, A.; Bensaude, O. An Evolutionary Conserved Hexim1 Peptide Binds to the Cdk9 Catalytic Site to Inhibit P-TEFb. Proc. Natl. Acad. Sci. 2016, 113 (45), 12721-12726.

https://doi.org/10.1073/pnas.1612331113.

(51) Binding of the 7SK SnRNA Turns the HEXIM1 Protein into a P-TEFb (CDK9/Cyclin T) Inhibitor. $E M B O$ J. 2004, 23 (13), 2608-2619. https://doi.org/10.1038/sj.emboj.7600275.

(52) Long, M. J. C.; Urul, D. A.; Aye, Y. Chapter Eleven - REX Technologies for Profiling and Decoding the Electrophile Signaling Axes Mediated by Rosetta Stone Proteins. In Methods in Enzymology; Shukla, A. K., Ed.; Academic Press, 2020; Vol. 633, pp 203-230. https://doi.org/10.1016/bs.mie.2019.02.039.

(53) Parvez, S.; Long, M. J.; Poganik, J. R.; Aye, Y. Redox Signaling by Reactive Electrophiles and Oxidants. Chem. Rev. 2018, 118 (18), 8798-8888.

(54) Parvez, S.; Fu, Y.; Li, J.; Long, M. J.; Lin, H.-Y.; Lee, D. K.; Hu, G. S.; Aye, Y. Substoichiometric Hydroxynonenylation of a Single Protein Recapitulates Whole-Cell-Stimulated Antioxidant Response. J. Am. Chem. Soc. 2015, 137 (1), 10-13.

(55) Weaver, D. S. The English Blunderbuss and Its Ballistics. Arms Armour 2020, 17 (2), $122-156$. https://doi.org/10.1080/17416124.2020.1727630.

(56) Kuljanin, M.; Mitchell, D. C.; Schweppe, D. K.; Gikandi, A. S.; Nusinow, D. P.; Bulloch, N. J.; Vinogradova, E. V.; Wilson, D. L.; Kool, E. T.; Mancias, J. D.; Cravatt, B. F.; Gygi, S. P.

Reimagining High-Throughput Profiling of Reactive Cysteines for Cell-Based Screening of Large Electrophile Libraries. Nat. Biotechnol. 2021, 39 (5), 630-641.

https://doi.org/10.1038/s41587-020-00778-3.

(57) Yang, F.; Gao, J.; Che, J.; Jia, G.; Wang, C. A Dimethyl-Labeling-Based Strategy for SiteSpecifically Quantitative Chemical Proteomics. Anal. Chem. 2018, 90 (15), 9576-9582. https://doi.org/10.1021/acs.analchem.8b02426.

(58) Weerapana, E.; Wang, C.; Simon, G. M.; Richter, F.; Khare, S.; Dillon, M. B. D.; Bachovchin, D. A.; Mowen, K.; Baker, D.; Cravatt, B. F. Quantitative Reactivity Profiling Predicts Functional Cysteines in Proteomes. Nature 2010, 468 (7325), 790-795.

https://doi.org/10.1038/nature09472.

(59) Parvez, S.; Long, M. J. C.; Lin, H.-Y.; Zhao, Y.; Haegele, J. A.; Pham, V. N.; Lee, D. K.; Aye, Y. TREX on-Demand Redox Targeting in Live Cells. Nat. Protoc. 2016, 11 (12), 2328-2356. https://doi.org/10.1038/nprot.2016.114.

(60) Parvez, S.; Long, M. J.; Lin, H.-Y.; Zhao, Y.; Haegele, J. A.; Pham, V. N.; Lee, D. K.; Aye, Y. T-REX on-Demand Redox Targeting in Live Cells. Nat. Protoc. 2016, 11 (12), 2328-2356.

(61) Long, M. J.; Parvez, S.; Zhao, Y.; Surya, S. L.; Wang, Y.; Zhang, S.; Aye, Y. Akt3 Is a Privileged First Responder in Isozyme-Specific Electrophile Response. Nat. Chem. Biol. 2017, 13 (3), 333338. 
(62) Zhao, Y.; Long, M. J.; Wang, Y.; Zhang, S.; Aye, Y. Ube2V2 Is a Rosetta Stone Bridging Redox and Ubiquitin Codes, Coordinating DNA Damage Responses. ACS Cent. Sci. 2018, 4 (2), 246259.

(63) Long, M. J. C.; Aye, Y. Privileged Electrophile Sensors: A Resource for Covalent Drug Development. Cell Chem. Biol. 2017, 24 (7), 787-800.

https://doi.org/10.1016/j.chembiol.2017.05.023.

(64) Butterfield, D. A.; Halliwell, B. Oxidative Stress, Dysfunctional Glucose Metabolism and Alzheimer Disease. Nat. Rev. Neurosci. 2019, 20 (3), 148-160. https://doi.org/10.1038/s41583-019-0132-6.

(65) Sirover, M. A. Pleiotropic Effects of Moonlighting Glyceraldehyde-3-Phosphate Dehydrogenase (GAPDH) in Cancer Progression, Invasiveness, and Metastases. Cancer Metastasis Rev. 2018, 37 (4), 665-676. https://doi.org/10.1007/s10555-018-9764-7.

(66) Hayes, J. D.; Dinkova-Kostova, A. T.; Tew, K. D. Oxidative Stress in Cancer. Cancer Cell 2020, 38 (2), 167-197. https://doi.org/10.1016/j.ccell.2020.06.001.

(67) Reva, B.; Antipin, Y.; Sander, C. Predicting the Functional Impact of Protein Mutations: Application to Cancer Genomics. Nucleic Acids Res. 2011, 39 (17), e118-e118. https://doi.org/10.1093/nar/gkr407.

(68) Cambronne, X. A.; Kraus, W. L. Location, Location, Location: Compartmentalization of NAD+ Synthesis and Functions in Mammalian Cells. Trends Biochem. Sci. 2020, 45 (10), 858-873. https://doi.org/10.1016/j.tibs.2020.05.010.

(69) Long, M. J. C.; Zhao, Y.; Aye, Y. Neighborhood Watch: Tools for Defining Locale-Dependent Subproteomes and Their Contextual Signaling Activities. RSC Chem. Biol. 2020, 1 (2), 42-55. https://doi.org/10.1039/D0CB00041H.

(70) Fu, Y.; Long, M. J. C.; Wisitpitthaya, S.; Inayat, H.; Pierpont, T. M.; Elsaid, I. M.; Bloom, J. C.; Ortega, J.; Weiss, R. S.; Aye, Y. Nuclear RNR- $\alpha$ Antagonizes Cell Proliferation by Directly Inhibiting ZRANB3. Nat. Chem. Biol. 2018, 14 (10), 943-954. https://doi.org/10.1038/s41589018-0113-5.

(71) Liu, X.; Long, M. J. C.; Hopkins, B. D.; Luo, C.; Wang, L.; Aye, Y. Precision Targeting of Pten-Null Triple-Negative Breast Tumors Guided by Electrophilic Metabolite Sensing. ACS Cent. Sci. 2020, 6 (6), 892-902. https://doi.org/10.1021/acscentsci.9b00893.

(72) Long, M. J. C. Time to Get Turned on by Chemical Biology. ChemBioChem 2021, 22 (5), 814817. https://doi.org/10.1002/cbic.202000497.

(73) Poganik, J. R.; Van Hall-Beauvais, A. K.; Long, M. J. C.; Disare, M. T.; Zhao, Y.; Aye, Y. The MRNA-Binding Protein HuR Is a Kinetically-Privileged Electrophile Sensor. Helv. Chim. Acta 2020, 103 (5), e2000041. https://doi.org/10.1002/hlca.202000041. 


\section{Figure 1}

\section{A}
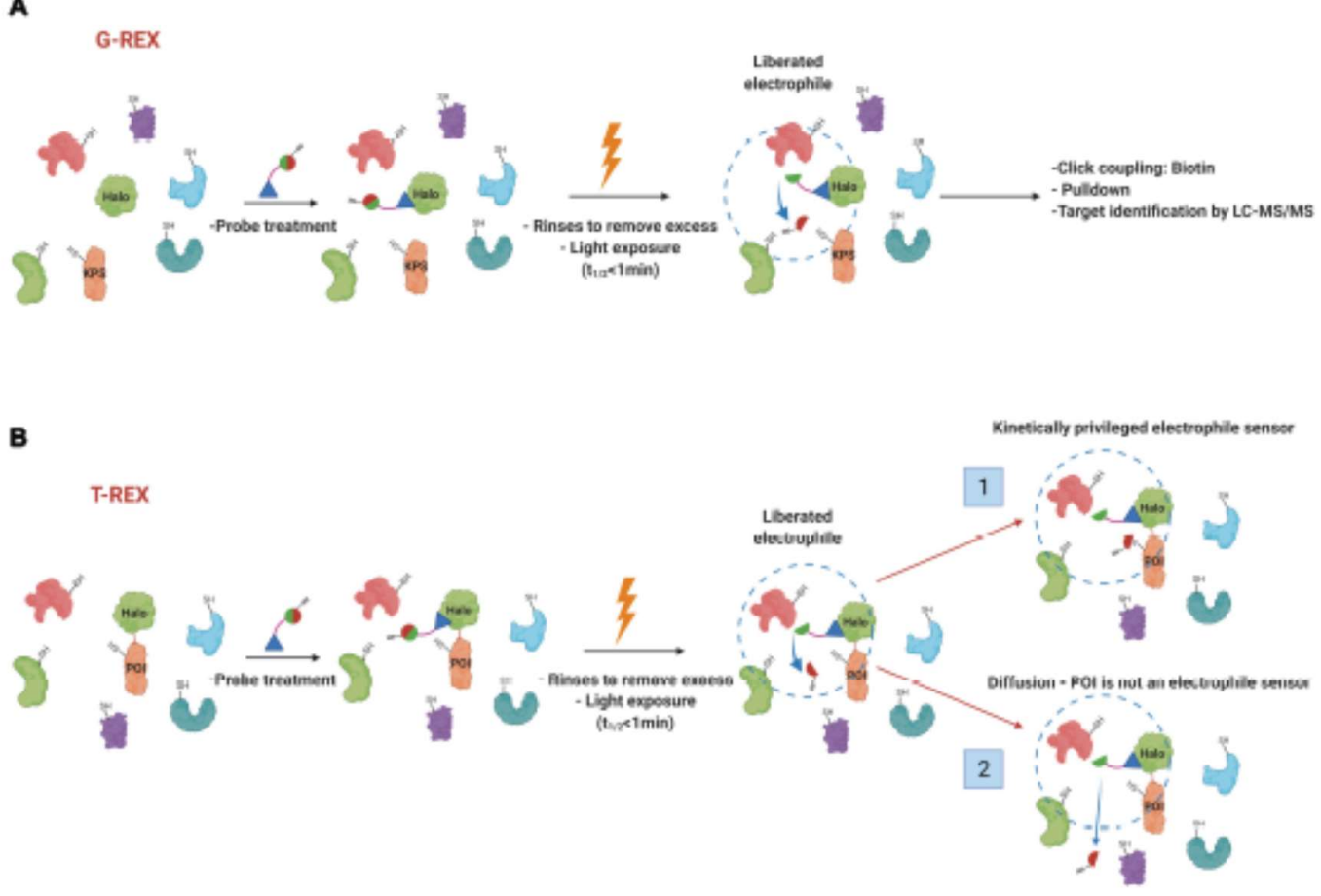

C


Figure 1. REX ${ }^{\mathrm{TM}}$ technologies. (A) Global reactive electrophiles and oxidants (G-REX ${ }^{\mathrm{TM}}$ ) profiling technique allows the identification of kinetically privileged electrophile sensor (KPS) proteins using bifunctional probes, light, and the haloalkane dehalogenase protein Tag (HaloTag). (B) Targetable reactive electrophiles and oxidants (T-REX ${ }^{\mathrm{TM}}$ ) allows single protein interrogation by introducing fusion 
proteins to HaloTag, typically separated by a Tobacco Etch Virus (TEV) protease recognition sequence. Two scenarios can occur upon the release of the electrophile in T-REX: 1) The released electrophile reacts with the protein of interest (POI); 2) The POI is not a sensor and the electrophile diffuses away. Typically good sensors of electrophiles have elevated labeling of the POI relative to insensitive POIs, which show no labeling. (C) HaloTag Pre-HNE (HtPreHNE) is an example of a bifunctional probe that binds covalently to HaloTag (Asp106) through the hexyl chloride motif (blue). Since REX technologies take place in live cells or animal models, rinses to remove the excess of unbound probe are necessary. The use of light $\left(365 \mathrm{~nm}, 5 \mathrm{~mW} / \mathrm{cm}^{2}\right)$ induces the photouncaging of HNE-alkyne (red) from the precursor molecule $\left(t_{1 / 2}<1 \mathrm{~min}\right)$. The liberated electrophile will then react in the close vicinity of the release site predominantly with kinetically privileged cysteine (Cys) residues. The liberated HNE-alkyne will then react with KPS proteins to generate Michael adducts. 
Figure 2

A<smiles>COC(=O)/C=C/C(=O)OC</smiles>

Dimethyl fumarate (Tecfidera)

FDA approved drug<smiles>O=C([O-])C=CC(=O)[O-]</smiles>

Fumarate

Native metabolite<smiles>CCCCCC(O)/C=C/C=O</smiles>

4-hydroxynonenal (4-HNE)

Native metabolite

Relapsing forms of MS

Involved in TCA and urea cycles

Lipid peroxidation product

B
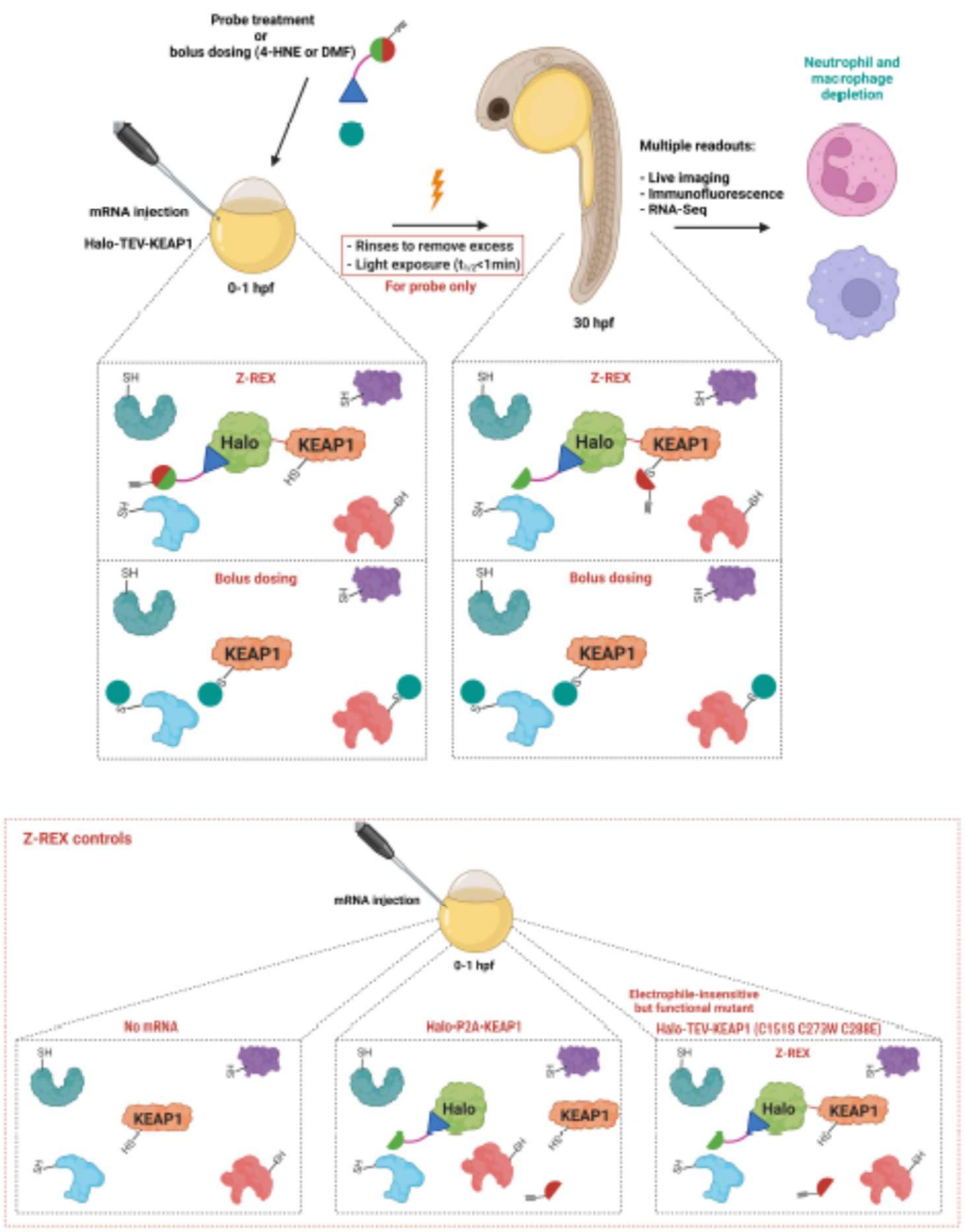

Figure 2. Tecfidera ${ }^{\circledR}$ activates neutrophil- and macrophage-specific apoptotic machinery. (A) Structures of dimethyl fumarate (DMF - Tecfidera ${ }^{\circledR}$ ), fumarate, and 4-hydroxynonenal (4-HNE). Tecfidera $^{\circledR}$ is an FDA-approved drug against relapsing forms of multiple sclerosis (MS), having high 
structural similarity to the native metabolite fumarate. 4-HNE is an example of a native reactive metabolite derived from lipid peroxidation, which is also an alpha, beta unsaturated-carbonyl compound. 4-HNE elicits the same phenotypic response in cells and animal models as Tecfidera ${ }^{\circledR}$ through the same signaling mechanism. (B) In zebrafish reactive electrophiles and oxidants (Z-REX ${ }^{\mathrm{TM}}$ ) profiling technology. mRNA is injected in zebrafish embryos $0-1$ hours post-fertilization (hpf) to express Halo-TEV-Keap1 with simultaneous treatment of HtPreHNE or vehicle. For the case of the probe-treated samples, $30 \mathrm{hpf}$ several washes are performed to remove excess compound prior to light exposure. Several assays have been coupled to Z-REX'M, including RNA sequencing in which transcripts associated with immune cells (neutrophil and macrophages) were downregulated in the treated samples compared to controls suggesting a link for the response on these particular cells. The use of transgenic zebrafish lines expressing fluorescent proteins under neutrophil- and macrophagespecific promoters has also been used to observe these cells' depletion by live imaging. Immunofluorescence has also been coupled to observe the activation of downstream regulators of the apoptosis pathway, such as Caspases.

There are three Z-REX controls for every experiment: 1) No mRNA, 2) mRNA injection for Halo-P2AKeap1 expression. The use of the P2A self-cleaving peptide allows expressing Halo independently from Keap1 to remove the proximity of the two proteins to show specific interaction of the liberated electrophile in the fusion construct, 3) mRNA injection for Halo-TEV-Keap1 (C151S C273W C288E) mutant expression. This mutant version of Keap1 is electrophile insensitive, but retains canonical Keap1 functions. 
A



B



Figure 3. Electrophile engagement with Keap1 can activate a cytoprotective or an apoptotic response in different cells types. (A) 4-HNE or DMF disrupt the Keap1-Nrf2-Wdr1 interaction inducing the translocation of Nrf2 into the nucleus and activation of the antioxidant response genes leading to an overall cytoprotective response. (B) In neutrophils and macrophages, Keap1 electrophile modification induces the disruption of the same complex. However, Keap1 has numerous other interacting partners, including Wdr1. This protein is also released from Keap1 upon electrophile labeling of Keap1. Released Wdr1 interacts with Cofilin, which leads to Bax translocation to the mitochondria. This 
process ultimately activates Caspase-9- and Caspase-3 the executioners of apoptosis. Native immune cells are highly susceptible to this pathway.

A

Cytoplasmic Localis-REX

Figure 4



B Nuclear Localis-REX
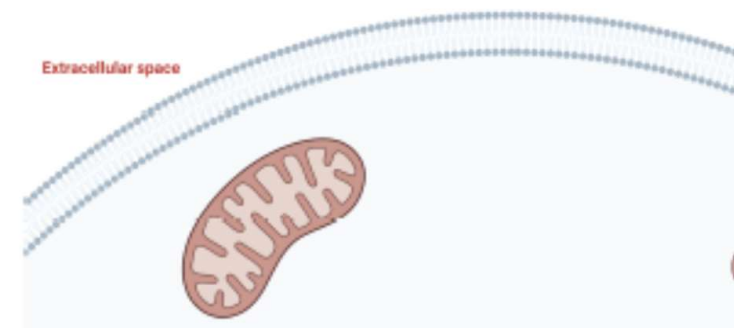

(n)


Figure 4. Locale-specific electrophilic-signal responsivity index (Localis-REX ${ }^{\mathrm{TM}}$ ) technology. By introducing localization sequences to the HaloTag protein, the G-REX technology can be repurposed to interrogate sensing within organellar proteomes. (A) Cytoplasmic Localis-REX ${ }^{\mathrm{TM}}$ has been established by introducing the outer mitochondrial membrane (MOM) localization sequence into HaloTag. (B) Nuclear Localis-REX ${ }^{\mathrm{TM}}$ was performed by using the nuclear localization sequence (NLS). 
Localis REX

Cytoplasmic CDK9 Localis-REX

\section{A}

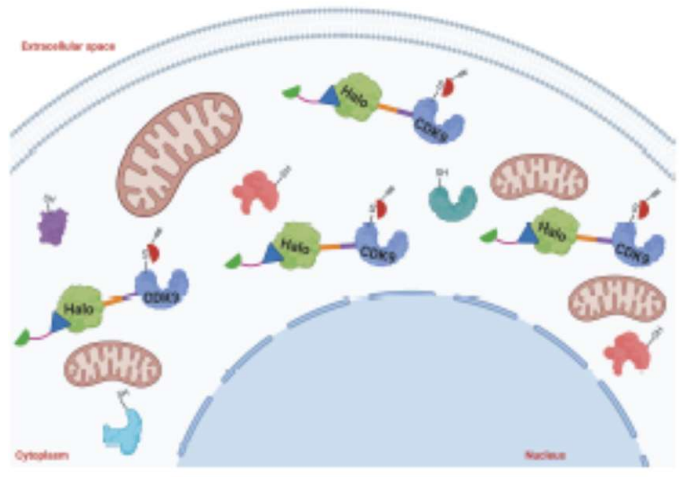

B
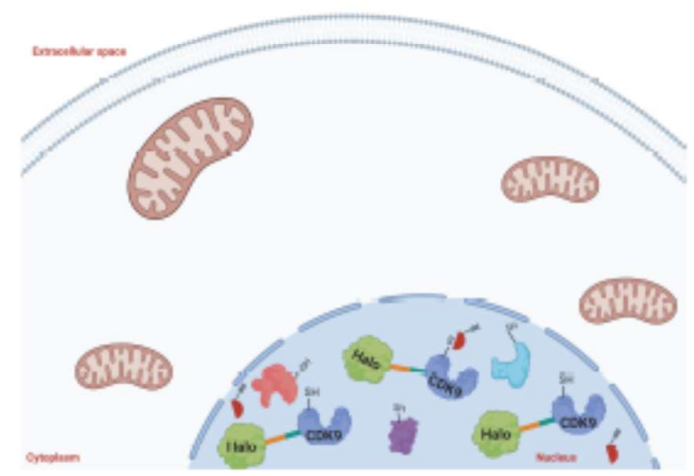
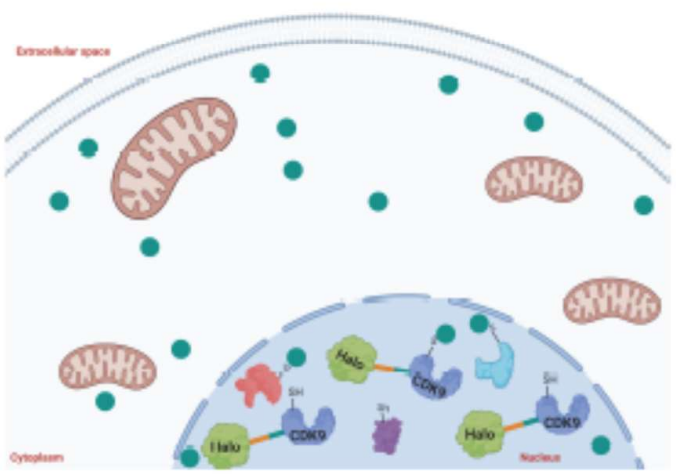

C

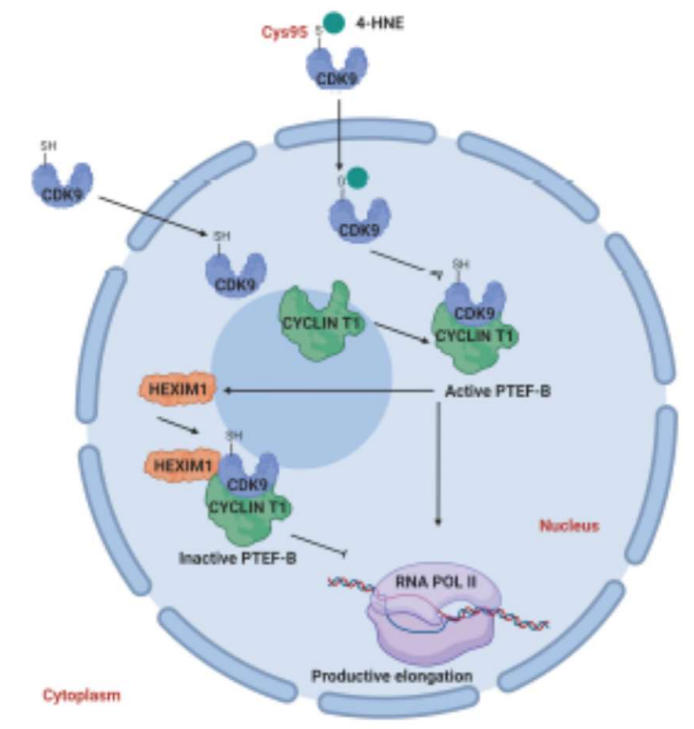

Figure 5. CDK9 was identified as a kinetically privileged electrophile sensor in the cytosol but not in its canonical locale, the nucleus. Localis-REX ${ }^{\mathrm{TM}}$ was combined with T-REX ${ }^{\mathrm{TM}}$ to introduce the localization tags in the fusion proteins and interrogate a specific $\mathrm{POI}$ in a locale-specific manner. (A) Using CDK9 
Localis-REX ${ }^{\mathrm{TM}}$ a higher electrophile sensitivity was observed when CDK9 was in the cytoplasm compared to the nucleus. CDK9 specific hydroxynonenylation occurs at Cys95 in the cytosol. After translocation of HNEylated CDK9 into the nucleus, the electrophilic modification in CDK9 impairs CDK9 complexation with CyclinT1 leading to overall transcriptional reduction.

Figure 6

A

Blunderbuss effect

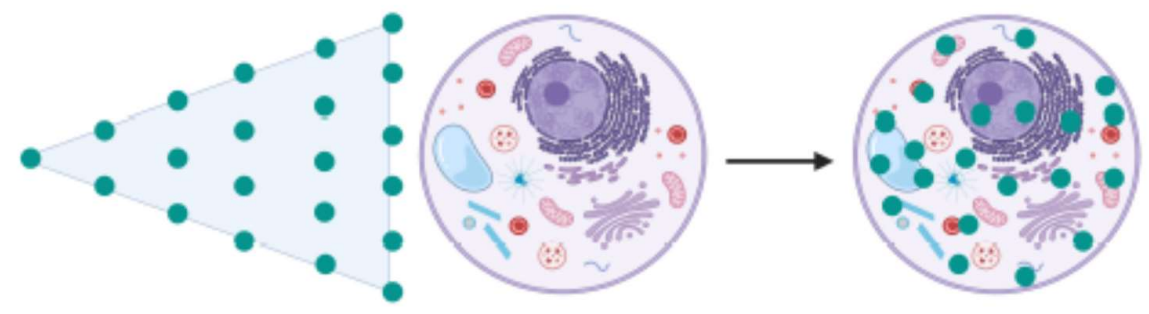

B

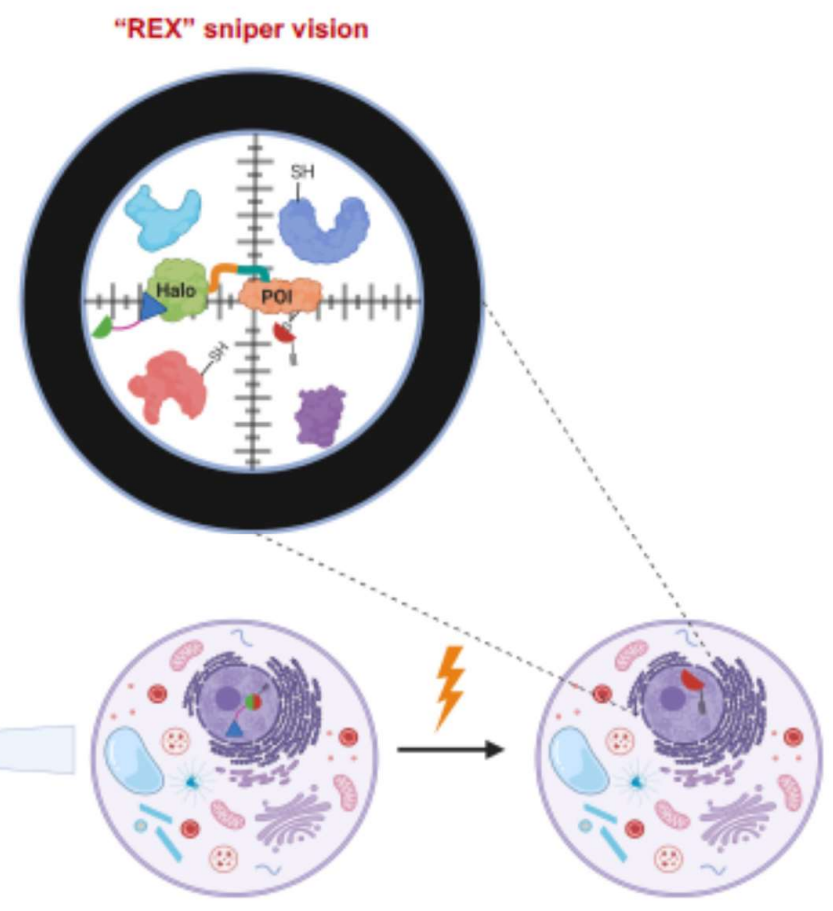

Figure 6. Blunderbuss effect vs. "REX" sniper vision. (A) Techniques preceding T-REX ${ }^{\mathrm{TM}}$ including the activity-based proteome profiling (ABPP) method, have relied on electrophile bolus dosing, which don't allow to study the proteome or an individual protein in a spatiotemporal manner. These techniques also induce undesired cellular effects due to multiple protein labeling, which often mask the real physiological electrophile effects making it difficult to study any signaling mechanisms. (B) REX $^{\mathrm{TM}}$ technologies have emerged to overcome these hurdles and confer a novel vision by introducing inert photocage probes that engage in a stoichiometric manner with HaloTag and releasing the reactive electrophiles on-demand upon light shining at particular locales. 


\section{Acknowledgements:}

Swiss National Science Funding (SNSF) Project Funding (310030_184729); NCCR Chemical Biology (SNSF); Swiss Federal Institute of Technology Lausanne (EPFL) (to Y.A.).

\section{Authors' brief biography:}

Marcus J C Long was born in Beverley. He was an undergraduate chemistry scholar of New College, Oxford University, and later went on to do a PhD in Biochemistry in Brandeis University. He has performed postdoctoral studies in chemical Biology in the Aye laboratory and in immunology in the Thome laboratory.

Pierre A Miranda $\mathbf{H}$ received his first master degree in chemical engineering at the University of Notre Dame, Indiana, USA. Subsequent research experience at the Mayo Clinic motivated him to pursue interdisciplinary research training. In 2020, he achieved his second master degree in chemical biology in the Aye laboratory, as a student of the NCCR chemical biology master research program jointly hosted by EPFL and UNIGE, Switzerland. Pierre is currently pursuing a doctoral degree at EPFL, under the guidance of Prof. Yimon Aye.

Yimon Aye read chemistry at Oxford University and completed her PhD and postdoctoral training in chemical and life sciences at Harvard University (with David Evans) and MIT (JoAnne Stubbe), respectively. She established her independent laboratory in mid-2012 and she is currently leading the Laboratory of Electrophiles And Genome Operation at EPFL, Switzerland. 